\title{
On s-normal Circulant and con-s-normal Circulant Matrices
}

\author{
K. Rajesh kannan ${ }^{18}$, N. Elumalai ${ }^{2}$, M. Kavitha ${ }^{3}$. \\ ${ }^{1}$ Department of Mathematics, A.V.C. College (Autonomous), Mannampandal, Tamil nadu, India \\ ${ }^{2}$ Department of Mathematics, A.V.C. College (Autonomous), Mannampandal, Tamil nadu, India \\ ${ }^{3}$ Department of Mathematics, Govt Arts college(Autonomous), Kumbakonam, Tamil nadu, India \\ Corresponding Author : rakkirajesh1986@gmail.com
}

Available online at: www.isroset.org

Received: 04/Sept/2018, Accepted: 05/Oct/2018, Online: 31/Oct/2018

\begin{abstract}
Circulant and con-s-normal circulant matrices are introduced and the basic roperties,results, consepts and structured theorems with examples are discussed in this paper
\end{abstract}

KEYWORDS: s-normal,con-s-normal,s-normaql circulant,con-s-normal circulant matrices.

\section{INTRODUCTION}

The concept of s-normal matrix, (conjugate) con-snormal matrix was introduced in [6], [7] and [8], some properties of s-normal matrix given in [1] .In this paper, our intention is to define s-normal circulant matrix, con-s normal circulant matrix also we discussed some properties and results on normal circulant matrix. Let $\mathrm{A}$ be circulant normal matrix, $\overline{\mathrm{A}}$ is called conjucate of $\mathrm{A}, \mathrm{A}^{\mathrm{T}}$ is called transpose of $\mathrm{A}, \mathrm{A}^{\mathrm{S}}$ is called secondary transpose of $\mathrm{A}, \mathrm{A}^{\ominus}$ is called conjugate secondary transpose of $A, A^{-1}$ is called inverse of $\mathrm{A}, \mathrm{A}^{\dagger}$ is called Moore Penrose of $\mathrm{A}$

\section{S-NORMAL CIRCULANT MATRICES}

\section{DEFINITION: 2.1}

For any given $c_{0}, c_{1}, c_{2}, \ldots c_{n-1} \in C^{n x n}$ the Circulant matrix $A=\left(A_{i, j}\right)_{n \times n}$ is defined by

$$
\begin{gathered}
\left(\mathrm{A}_{\mathrm{i}, \mathrm{j}}\right)=\mathrm{A}_{\mathrm{j}-1(\bmod \mathrm{n})} \\
A=\left[\begin{array}{ccccc}
c_{0} & c_{1} & c_{2} & \ldots & c_{n-1} \\
c_{n-1} & c_{0} & c_{1} & \ldots & c_{n-2} \\
c_{n-2} & c_{n-1} & c_{0} & \ldots & c_{n-3} \\
\vdots & \vdots & \vdots & \vdots & \vdots \\
c_{1} & c_{2} & c_{3} & \ldots & c_{0}
\end{array}\right]
\end{gathered}
$$

\section{DEFINITION: 2.2}

A Circulant matrix $A \in C^{\mathrm{nx}}$ is said to be normal Circulant matrix if $\mathrm{AA}^{*}=\mathrm{A}^{*} \mathrm{~A}$
DEFINITION: $\mathbf{2 . 3}$

A matrix $A \in C^{n \times n}$ is said to be s-normal Circulant matrix if $A A^{\Theta}=A^{\Theta} A$ where $A^{\ominus}=\overline{A^{s}}$

EXAMPLE: 2.4

$$
\begin{aligned}
& \mathrm{A}=\left[\begin{array}{ccc}
2 & 1+i & 1-i \\
1-i & 2 & 1+i \\
1+i & 1-i & 2
\end{array}\right] \\
& \mathrm{A} \mathrm{A}^{\Theta}=\left[\begin{array}{lll}
4 & 6 & 6 \\
6 & 4 & 6 \\
6 & 6 & 4
\end{array}\right]=\mathrm{A}^{\ominus} \mathrm{A}
\end{aligned}
$$

\section{RESULT:2.5}

(i) If $\mathrm{A}$ is Circulant matrix then $\mathrm{A}=\mathrm{A}^{\mathrm{S}}$

(ii) Let a Circulant matrix $A \in C^{n \times n}$ is said to be s-normal Circulant matrix if $\mathrm{A} \overline{\mathrm{A}}=\overline{\mathrm{A}} \mathrm{A}\left(\because \mathrm{A}=\mathrm{A}^{\mathrm{S}}\right)$

\section{THEOREM: 2.6}

Let $\mathrm{A}, \mathrm{B} \in \mathrm{C}^{\mathrm{nx} \mathrm{n}}$ are s-normal circulant matrices then $A \pm B$ is also s-normal circulant matrices.

Proof:

Let $\mathrm{A}, \mathrm{B}$ are s-normal circulant matrices then $\mathrm{AA}^{\ominus}=\mathrm{AA}$ and $\mathrm{BB}^{\Theta}=\mathrm{B}^{\Theta} \mathrm{B}$

To prove $\mathrm{A} \pm \mathrm{B}$ are s-normal circulant matrices. We will show that $(\mathrm{A} \pm \mathrm{B})(\mathrm{A} \pm \mathrm{B})^{\Theta}=(\mathrm{A} \pm \mathrm{B})^{\Theta}(\mathrm{A} \pm \mathrm{B})$

$$
\text { Now } \begin{aligned}
(A \pm B)(A \pm B)^{\Theta}= & (A \pm B)\left(A^{\Theta} \pm B^{\Theta}\right) \\
& =(A \pm B)\left(A^{\Theta} \pm B^{\Theta}\right)
\end{aligned}
$$




$$
\begin{aligned}
& =\mathrm{AA}^{\Theta} \pm \mathrm{BA}^{\Theta} \pm \mathrm{AB}^{\Theta} \pm \mathrm{BB}^{\Theta} \\
& =\mathrm{A}^{\Theta} \mathrm{A} \pm \mathrm{B}^{\Theta} \mathrm{A} \pm \mathrm{A}^{\Theta} \mathrm{B} \pm \mathrm{B}^{\Theta} \mathrm{B} \\
& =\left(\mathrm{A}^{\Theta} \pm \mathrm{B}^{\Theta}\right) \mathrm{A} \pm\left(\mathrm{A}^{\Theta} \pm \mathrm{B}^{\Theta}\right) \mathrm{B} \\
& =\left(\mathrm{A}^{\Theta} \pm \mathrm{B}^{\Theta}\right)(\mathrm{A} \pm \mathrm{B}) \\
& =(\mathrm{A} \pm \mathrm{B})^{\ominus}(\mathrm{A} \pm \mathrm{B})
\end{aligned}
$$

Therefore $\mathrm{A} \pm \mathrm{B}$ are s-normal circulant matrices.

\section{THEOREM: 2.7}

Let $\mathrm{A}, \mathrm{B} \in \mathrm{C}^{\mathrm{nx} \mathrm{n}}$ are s-normal circulant matrices and $\mathrm{AB}=\mathrm{BA}$ then $\mathrm{AB}$ is also s-normal circulant matrix

Proof:

Let $\mathrm{A}, \mathrm{B}$ are s-normal circulant matrices then $\mathrm{AA}^{\Theta}=\mathrm{A}^{\Theta} \mathrm{A}$ and $\mathrm{BB}^{\Theta}=\mathrm{B}^{\Theta} \mathrm{B}$ given $\mathrm{AB}=\mathrm{BA}$

To prove $A B$ is s-normal circulant matrix. We will show that $(\mathrm{AB})(\mathrm{AB})^{\Theta}=(\mathrm{AB})^{\Theta}(\mathrm{AB})$

$$
\text { Now } \begin{aligned}
(\mathrm{AB})(\mathrm{AB})^{\ominus}= & \mathrm{AB} \mathrm{A}^{\ominus} \mathrm{B}^{\Theta} \\
& =\mathrm{BA}^{\Theta} \mathrm{B}^{\Theta} \\
& =\mathrm{BA}^{\Theta} \mathrm{AB}^{\Theta} \\
& =\mathrm{BA}^{\ominus} \mathrm{B}^{\Theta} \mathrm{A} \\
& =\mathrm{A}^{\ominus} \mathrm{BB}^{\ominus} \mathrm{A} \\
& =\mathrm{A}^{\ominus} \mathrm{B}^{\Theta} \mathrm{BA} \\
& =(\mathrm{AB})^{\ominus}(\mathrm{AB})
\end{aligned}
$$

Therefore AB is s-normal circulant matrix.

\section{THEOREM: 2.8}

Let $\mathrm{A}, \mathrm{B} \in \mathrm{C}^{\mathrm{nx} \mathrm{n}}$ are s-normal circulant matrices and $\mathrm{AB}=\mathrm{BA}$ then $\mathrm{AB}^{\Theta}$ and $\mathrm{A}^{\ominus} \mathrm{B}$ are also s-normal circulant matrices.

\section{Proof:}

Let $\mathrm{A}, \mathrm{B}$ are s-normal circulant matrices then $\mathrm{AA}^{\Theta}=\mathrm{A}^{\Theta} \mathrm{A}$ and $\mathrm{BB}^{\ominus}=\mathrm{B}^{\ominus} \mathrm{B}$ given $\mathrm{AB}=\mathrm{BA}$

To prove $\mathrm{AB}^{\Theta}$ is s-normal circulant matrix. We will show that $\left(\mathrm{AB}^{\Theta}\right)\left(\mathrm{AB}^{\Theta}\right)^{\Theta}=\left(\mathrm{AB}^{\Theta}\right)^{\Theta}\left(\mathrm{AB}^{\Theta}\right)$

$\operatorname{Now}(A B)(A B)^{\ominus}=(A B)^{\Theta}(A B)$

$$
\begin{aligned}
& A B B^{\ominus} A^{\Theta}=(B A)^{\ominus}(B A) \quad \text { Where } A B=B A \\
& A B B^{\ominus} A^{\Theta}=A^{\ominus} B^{\ominus} B A
\end{aligned}
$$

$$
\begin{aligned}
\mathrm{AB}^{\Theta} \mathrm{B} \mathrm{A}^{\Theta} & =\mathrm{A}^{\Theta} \mathrm{BB}^{\Theta} \quad \text { Where } \mathrm{B}^{\Theta} \mathrm{B}=\mathrm{BB}^{\Theta} \\
\mathrm{AB}^{\Theta}\left(\mathrm{B}^{\Theta}\right)^{\Theta} \mathrm{A}^{\Theta} & =\mathrm{A}^{\Theta}\left(\mathrm{B}^{\Theta}\right)^{\Theta} \mathrm{B}^{\Theta} \mathrm{A} \quad \text { Where }\left(\mathrm{B}^{\Theta}\right)^{\Theta}=\mathrm{B} \\
\left(\mathrm{AB}^{\Theta}\right)\left(\mathrm{AB}^{\Theta}\right)^{\Theta} & =\left(\mathrm{AB}^{\Theta}\right)^{\Theta}\left(\mathrm{AB}^{\Theta}\right)
\end{aligned}
$$

Therefore $\mathrm{AB}^{\Theta}$ is s-normal circulant matrices.

Similarly we can prove $\mathrm{A}^{\ominus} \mathrm{B}$ is s-normal circulant matrices.

\section{THEOREM: 2.9}

Let $\mathrm{A} \in \mathrm{C}^{\mathrm{nx} \mathrm{n}}$ be s-normal circulant matrix then

(i) iA is s-normal circulant matrix

(ii) $\quad-\mathrm{iA}$ is s-normal circulant matrix

Proof:

$\mathrm{AA}^{\Theta}=\mathrm{A}^{\Theta} \mathrm{A}$

Let $\mathrm{A}$ be a s-normal circulant matrix then

To prove (i) iA is s-normal circulant matrix. We will show that $(\mathrm{iA})(\mathrm{iA})^{\Theta}=(\mathrm{iA})^{\Theta}(\mathrm{iA})$

Now $\mathrm{AA}^{\ominus}=\mathrm{A}^{\Theta} \mathrm{A}$

$$
\begin{aligned}
-i^{2} \mathrm{AA}^{\Theta} & =-\mathrm{i}^{2} \mathrm{~A}^{\Theta} \mathrm{A} \\
(\mathrm{iA})(-\mathrm{i}) \mathrm{A}^{\Theta} & =(-\mathrm{i}) \mathrm{A}^{\Theta}(\mathrm{iA}) \\
(\mathrm{iA})(\bar{i}) \mathrm{A}^{\Theta} & =(\bar{i}) \mathrm{A}^{\Theta}(\mathrm{iA}) \text { where }(-\mathrm{i})=\overline{\mathrm{i}} \\
(\mathrm{iA})(\overline{\boldsymbol{i}})^{\mathrm{S}} \mathrm{A}^{\Theta} & =(\bar{i})^{\mathrm{S}} \mathrm{A}^{\Theta}(\mathrm{iA}) \text { where }(\bar{i})^{\mathrm{S}}=\overline{\mathrm{i}} \\
(\mathrm{iA}) \mathrm{i}^{\Theta} \mathrm{A}^{\Theta} & =\mathrm{i}^{\Theta} \mathrm{A}^{\Theta}(\mathrm{iA}) \text { where } \mathrm{i}^{\Theta}=(\overline{\mathrm{i}})^{\mathrm{S}} \\
(\mathrm{iA})(\mathrm{iA})^{\Theta} & =(\mathrm{iA})^{\Theta}(\mathrm{iA})
\end{aligned}
$$

Therefore iA is s-normal circulant matrix.

(ii) -iA is s-normal circulant matrix. We will show that (iA) $(-\mathrm{iA})^{\Theta}=(-\mathrm{iA})^{\Theta}(-\mathrm{iA})$

Now $\mathrm{AA}^{\Theta}=\mathrm{A}^{\Theta} \mathrm{A}$

$$
-i^{2} A A^{\Theta}=-i^{2} A^{\Theta} A
$$

(-i) i A $A^{\ominus}=$ i (-i) $A^{\ominus} A$

$(-\mathrm{i} A)(\mathrm{i}) \mathrm{A}^{\Theta}=(\mathrm{i}) \mathrm{A}^{\Theta}(-\mathrm{i} \mathrm{A})$

$(-\mathrm{i} \mathrm{A})(\overline{-i}) \mathrm{A}^{\Theta}=(\overline{-i}) \mathrm{A}^{\Theta}(-\mathrm{i} \mathrm{A})$ where $\mathrm{i}=\overline{-i}$

$(-\mathrm{iA})(\overline{-i})^{\mathrm{S}} \mathrm{A}^{\ominus}=(\overline{-i})^{\mathrm{S}} \mathrm{A}^{\ominus}(-\mathrm{i} \mathrm{A})$

$(-\mathrm{i} A)-i^{\ominus} A^{\Theta}=-i^{\Theta} A^{\Theta}(-i \mathrm{~A})$ where $-i^{\Theta}=(\overline{-i})^{S}$

$(-\mathrm{iA})(-\mathrm{iA})^{\ominus}=(-\mathrm{iA})^{\ominus}(-\mathrm{iA})$ 
Therefore -iA is s-normal circulant matrix.

\section{THEOREM: 2.9}

Let $\mathrm{A} \in \mathrm{C}^{\mathrm{nx} \mathrm{n}}$ are s-normal circulant matrix then

(i) $\bar{A}$ is s-normal circulant matrix

(ii) $\mathrm{A}^{\mathrm{S}}$ is s-normal circulant matrix

(iii) $\mathrm{A}^{\ominus}$ is s-normal circulant matrix

(iv) $\lambda \mathrm{A}$ is s-normal circulant matrix (Where $\lambda$ is a non zero real no)

\section{Proof:}

Let $\mathrm{A}$ be a s-normal circulant matrix then $\mathrm{AA}^{\Theta}=\mathrm{A}^{\Theta} \mathrm{A}$

Proof of $(i) \mathrm{AA}^{\ominus}=\mathrm{A}^{\ominus} \mathrm{A}$

$$
\begin{gathered}
\overline{\mathrm{AA}^{\theta}}=\overline{\mathrm{AA}^{\theta}} \\
\overline{\mathrm{A}^{\theta}} \overline{\mathrm{A}}=\overline{\mathrm{A}} \overline{\mathrm{A}^{\theta}} \\
\overline{(A)}^{\ominus} \bar{A}=\bar{A} \overline{(A)}^{\ominus} \\
\bar{A} \overline{(A)}^{\ominus}=\overline{(A)}^{\ominus} \bar{A}
\end{gathered}
$$

Therefore $\bar{A}$ is s-normal circulant matrix

Proof of (ii) $\mathrm{AA}^{\Theta}=\mathrm{A}^{\Theta} \mathrm{A}$

$$
\begin{aligned}
& \left(A A^{\Theta}\right)^{S}=\left(A^{\Theta} A\right)^{S} \\
& \left(A^{\Theta}\right)^{S} A^{S}=(A)^{S}\left(A^{\Theta}\right)^{S} \\
& \left(A^{S}\right)^{\Theta} A^{S}=(A)^{S}\left(A^{S}\right)^{\Theta} \\
& (A)^{S}\left(A^{S}\right)^{\Theta}=\left(A^{S}\right)^{\Theta} A^{S}
\end{aligned}
$$

Therefore $\mathrm{A}^{\mathrm{S}}$ is s-normal circulant matrix

Proof of (iii) $\mathrm{AA}^{\Theta}=\mathrm{A}^{\Theta} \mathrm{A}$

$$
\begin{aligned}
& \left(A A^{\Theta}\right)^{\Theta}=\left(A^{\Theta} A\right)^{\Theta} \\
& \left(A^{\Theta}\right)^{\Theta} A^{\Theta}=A^{\Theta}\left(A^{\Theta}\right)^{\Theta} \\
& A^{\Theta}\left(A^{\Theta}\right)^{\Theta}=\left(A^{\Theta}\right)^{\Theta} A^{\Theta}
\end{aligned}
$$

Therefore $\mathrm{A}^{\Theta}$ is s-normal circulant matrix

Proof of (iv) $\mathrm{AA}^{\Theta}=\mathrm{A}^{\Theta} \mathrm{A}$

$$
\lambda^{2} \mathrm{AA}^{\Theta}=\lambda^{2} \mathrm{~A}^{\Theta} \mathrm{A}
$$

$$
(\lambda \mathrm{A})(\lambda \mathrm{A})^{\Theta}=(\lambda \mathrm{A})^{\Theta}(\lambda \mathrm{A}) \text { where } \lambda=\lambda^{\Theta}
$$

Therefore $\lambda \mathrm{A}$ is s-normal circulant matrix
THEOREM: 2.10

Let $A \in C^{n x n}$ and $A^{-1}$ be an inverse of $A$ then $A$ is s-normal circulant matrix iff $\mathrm{A}^{-1}$ is s-normal circulant matrix

Proof: $\mathrm{A}^{\ominus} \mathrm{A}$

Let $\mathrm{A}$ be a s-normal circulant matrix then $\mathrm{AA}^{\ominus}=$

To prove $\mathrm{A}^{-1}$ is $\mathrm{k}$-normal circulant matrix. We will show that $\left(\mathrm{A}^{-1}\right)\left(\mathrm{A}^{-1}\right)^{\Theta}=\left(\mathrm{A}^{-1}\right)^{\Theta}\left(\mathrm{A}^{-1}\right)$

Now

$$
A A^{\ominus}=A^{\Theta} A
$$$$
\left(\mathrm{AA}^{\Theta}\right)^{-1}=\left(\mathrm{A}^{\Theta} \mathrm{A}\right)^{-1}
$$$$
\left(A^{\Theta}\right)^{-1} A^{-1}=A^{-1}\left(A^{\Theta}\right)^{-1}
$$$$
\left(A^{\Theta}\right)^{-1} A^{-1}=A^{-1}\left(A^{\Theta}\right)^{-1}
$$$$
\left(\mathrm{A}^{-1}\right)^{\Theta} \mathrm{A}^{-1}=\mathrm{A}^{-1}\left(\mathrm{~A}^{-1}\right)^{\Theta}
$$$$
\mathrm{A}^{-1}\left(\mathrm{~A}^{-1}\right)^{\Theta}=\left(\mathrm{A}^{-1}\right)^{\Theta} \mathrm{A}^{-1}
$$

Therefore $\mathrm{A}^{-1}$ is s-normal circulant matrix.

Let as assume that, $\mathrm{A}^{-1}$ is s-normal circulant matrix.

To prove A is s-normal circulant matrix. We will show that $\mathrm{A} \mathrm{A}^{\Theta}=\mathrm{A} \mathrm{A}^{\Theta}$

Now

$$
\begin{aligned}
A^{-1}\left(A^{-1}\right)^{\Theta} & =\left(A^{-1}\right)^{\Theta} A^{-1} \\
\left(A^{-1}\left(A^{-1}\right)^{\Theta}\right)^{-1} & =\left(\left(A^{-1}\right)^{\Theta} A^{-1}\right)^{-1} \\
A^{\Theta}\left(A^{-1}\right)^{-1} & =\left(A^{-1}\right)^{-1} A^{\Theta} \\
A^{\ominus} A & =A A^{\Theta} \\
A A^{\Theta} & =A^{\ominus} A
\end{aligned}
$$

Therefore A is s-normal circulant matrix

\section{THEOREM: 2.11}

Let $A \in C^{n x n}$ and $A^{\dagger}$ be the Moore Penrose inverse of $A$ then $\mathrm{A}$ is s-normal circulant matrix iff $\mathrm{A}^{\dagger}$ is s-normal circulant matrix

Proof:

$\mathrm{AA}^{\ominus}=\mathrm{A}^{\Theta} \mathrm{A}$

Let $\mathrm{A}$ be a s-normal circulant matrix then

To prove $A^{\dagger}$ is s-normal circulant matrix. We will show that $\left(A^{f}\right)\left(A^{\dagger}\right)^{\Theta}=\left(A^{\dagger}\right)^{\Theta}\left(A^{\dagger}\right)$

Now

$$
\mathrm{A} \mathrm{A}^{\Theta}=\mathrm{A}^{\Theta} \mathrm{A}
$$

$$
\left(\mathrm{AA}^{\Theta}\right)^{\dagger}=\left(\mathrm{A}^{\Theta} \mathrm{A}\right)^{\dagger}
$$

$$
\left(A^{\ominus}\right)^{\dagger} A^{\dagger}=A^{\dagger}\left(A^{\ominus}\right)^{\dagger}
$$




$$
\begin{aligned}
& \left(A^{\dagger}\right)^{\Theta} A^{\dagger}=A^{\dagger}\left(A^{\dagger}\right)^{\Theta} \\
& A^{\dagger}\left(A^{\dagger}\right)^{\Theta}=\left(A^{\dagger}\right)^{\Theta} A^{\dagger}
\end{aligned}
$$

Therefore $\mathrm{A}^{\dagger}$ is s-normal circulant matrix.

Let as assume that, $\mathrm{A}^{\dagger}$ is s-normal circulant matrix.

To prove $\mathrm{A}$ is s-normal circulant matrix. We will show that $\mathrm{A} \mathrm{A}^{\Theta}=\mathrm{A} \mathrm{A}^{\Theta}$

Now

$$
\begin{aligned}
A^{\dagger}\left(A^{\dagger}\right)^{\Theta} & =\left(A^{\dagger}\right)^{\Theta} A^{\dagger} \\
\left(A^{\dagger}\left(A^{\dagger}\right)^{\ominus}\right)^{\dagger} & =\left(\left(A^{\dagger}\right)^{\Theta} A^{\dagger}\right)^{\dagger} \\
A^{\Theta}\left(A^{\dagger}\right)^{\dagger} & =\left(A^{\dagger}\right)^{\dagger} A^{\Theta} \\
A^{\ominus} A & =A A^{\Theta} \\
A A^{\Theta} & =A^{\ominus} A
\end{aligned}
$$

Therefore A is s-normal circulant matrix.

\section{CONJUGATE S-NORMAL CIRCULANT MATRICES}

\section{DEFINITION: 3.1}

A Circulant matrix $A \in C^{n \times n}$ is said to be con k-normal Circulant matrix if $\mathrm{AA}^{\Theta}=\mathrm{A}^{\mathrm{S}} \bar{A}$

\section{EXAMPLE: 3.2}

$$
\begin{aligned}
\mathrm{A} & =\left[\begin{array}{ccc}
2 & 1+i & 1-i \\
1-i & 2 & 1+i \\
1+i & 1-i & 2
\end{array}\right] \\
\mathrm{A} \mathrm{A}^{\ominus} & =\left[\begin{array}{lll}
4 & 6 & 6 \\
6 & 4 & 6 \\
6 & 6 & 4
\end{array}\right]=\mathrm{A}^{\mathrm{S}} \bar{A}
\end{aligned}
$$

\section{THEOREM: 3.3}

Let $A, B \in C^{n \times n}$ are con s-normal circulant matrices then $\mathrm{A} \pm \mathrm{B}$ are also con s-normal circulant matrices.

\section{Proof:}

Let $\mathrm{A}, \mathrm{B}$ are con s-normal circulant matrices then $\mathrm{AA}^{\theta}=$ $\mathrm{A}^{\mathrm{S}} \bar{A}$ and $\mathrm{BB}^{\Theta}=\mathrm{B}^{\mathrm{S}} \bar{B}$

To prove $\mathrm{A} \pm \mathrm{B}$ are con s-normal circulant matrices.

We will show that $(\mathrm{A} \pm \mathrm{B})(\mathrm{A} \pm \mathrm{B})^{\Theta}=(\mathrm{A} \pm \mathrm{B})^{\mathrm{S}} \overline{(A \pm B)}$.

$$
\text { Now } \begin{aligned}
(A \pm B)(A \pm B)^{\Theta}= & (A \pm B)\left(A^{\ominus} \pm B^{\Theta}\right) \\
& =(A \pm B)\left(A^{\Theta} \pm B^{\ominus}\right) \\
& =A A^{\Theta} \pm \mathrm{AB}^{\Theta} \pm \mathrm{BA}^{\Theta} \pm \mathrm{BB}^{\Theta}
\end{aligned}
$$

$$
\begin{aligned}
& =\mathrm{A}^{\mathrm{S}} \bar{A} \pm \mathrm{B}^{\mathrm{S}} \bar{A} \pm \mathrm{A}^{\mathrm{S}} \bar{B} \pm \mathrm{B}^{\mathrm{S}} \bar{B} \\
& =\mathrm{A}^{\mathrm{S}}[\overline{A \pm B}] \pm \mathrm{B}^{\mathrm{S}}[\overline{A \pm B}] \\
& =\left[\mathrm{A}^{\mathrm{S}} \pm \mathrm{B}^{\mathrm{S}}\right][\overline{A \pm B}] \\
& =[\mathrm{A} \pm \mathrm{B}]^{\mathrm{S}}[\overline{A \pm B}] \quad \text { Where }[\mathrm{A} \pm
\end{aligned}
$$$$
\mathrm{B}]^{\mathrm{S}}=\left[\mathrm{A}^{\mathrm{S}} \pm \mathrm{B}^{\mathrm{S}}\right],[\overline{A \pm B}]=\bar{A} \pm \bar{B}
$$

\section{THEOREM: 3.4}

Let $\mathrm{A}, \mathrm{B} \in \mathrm{C}^{\mathrm{nx} \mathrm{n}}$ are con s-normal circulant matrices and $\mathrm{AB}=\mathrm{BA}$ then $\mathrm{AB}$ is also con s-normal circulant matrix Proof:

Let $\mathrm{A}, \mathrm{B}$ are con s-normal circulant matrices then $\mathrm{AA}^{\Theta}=\mathrm{A}^{\mathrm{S}} \bar{A}$ and $\mathrm{BB}^{\Theta}=\mathrm{B}^{\mathrm{S}} \bar{B}$

To prove $\mathrm{AB}$ is con s-normal circulant matrix. We will show that $(\mathrm{AB})(\mathrm{AB})^{\Theta}=(\mathrm{AB})^{\mathrm{S}} \overline{(A B)}$

$$
\begin{aligned}
\text { Now }(\mathrm{AB})(\mathrm{AB})^{\Theta}= & \mathrm{A} \mathrm{B}^{\Theta} \mathrm{B}^{\Theta} \mathrm{A}^{\Theta} \\
& =\left(\mathrm{AB}^{\Theta}\right) \mathrm{B}^{\Theta} \\
= & \left(\mathrm{AB}^{\Theta}\right) \mathrm{A}^{\mathrm{S}} \bar{B} \\
& =\left(\mathrm{AB}^{\Theta}\right) \mathrm{A}^{\mathrm{S}} \bar{B} \\
& =\mathrm{B}^{\mathrm{S}} \bar{A} \mathrm{~A}^{\mathrm{S}} \bar{B} \\
& =\mathrm{B}^{\mathrm{S}} \mathrm{A}^{\mathrm{S}} \bar{A} \bar{B}
\end{aligned}
$$

\section{THEOREM: 3.5}

Let $A \in C^{n x n}$ be con s-normal circulant matrix then

(i) $\quad \mathrm{iA}$ is con s-normal circulant matrix

(ii) $\quad-\mathrm{iA}$ is con s-normal circulant matrix

Proof:

$\mathrm{A}^{\mathrm{S}} \bar{A}$

Let $\mathrm{A}$ is con s-normal circulant matrix then $\mathrm{AA}^{\Theta}=$

To prove (i) iA is con s-normal circulant matrix. We will show that $(\mathrm{iA})(\mathrm{iA})^{\Theta}=(\mathrm{iA})^{\mathrm{S}} \overline{(\mathrm{iA})}$

Now $\mathrm{AA}^{\Theta}=\mathrm{A}^{\mathrm{s}} \bar{A}$

$$
\mathrm{i}^{2} \mathrm{AA}^{\Theta}=\left(\mathrm{i}^{2}\right) \mathrm{A}^{\mathrm{S}} \bar{A}
$$

(iA) $\left(i A^{\ominus}\right)=(i) A^{\mathrm{S}}(\mathrm{i} \bar{A})$

(iA) $(-\mathrm{i})^{\Theta} \mathrm{A}^{\Theta}=\left(\mathrm{i}^{\mathrm{S}}\right) \mathrm{A}^{\mathrm{S}}(-\overline{i A})$

$-(\mathrm{iA})(\mathrm{iA})^{\ominus}=-(\mathrm{iA})^{\mathrm{S}}(\overline{i A})$

$(\mathrm{iA})(\mathrm{iA})^{\Theta}=(\mathrm{iA})^{\mathrm{S}}(\overline{i A})$ 
Therefore iA is con s-normal circulant matrix.

(ii) - $\mathrm{iA}$ is con s-normal circulant matrix. We will show that $(-\mathrm{iA})(-\mathrm{iA})^{\Theta}=(-\mathrm{iA})^{\mathrm{S}}(-\overline{i A})$

$$
\begin{aligned}
& \text { Now } \mathrm{AA}^{\Theta}=\mathrm{A}^{\mathrm{S}} \bar{A} \\
& -\mathrm{i}^{2} \mathrm{AA}^{\Theta}=(-\mathrm{i})^{2} \mathrm{~A}^{\mathrm{S}} \bar{A} \\
& (-\mathrm{iA})\left(\mathrm{iA}^{\Theta}\right)=\left(-\mathrm{i}^{\mathrm{S}}\right)(\mathrm{i} \bar{A}) \\
& (-\mathrm{iA})\left(-\mathrm{i}^{\Theta} \mathrm{A}^{\Theta}\right)=\left(-\mathrm{i}^{\mathrm{S}} \mathrm{A}^{\mathrm{S}}\right)(-\overline{i A}) \\
& (-\mathrm{iA})(-\mathrm{iA})^{\Theta}=(-\mathrm{iA})^{\mathrm{S}}(-\overline{i A})
\end{aligned}
$$

Therefore -iA is con s-normal circulant matrix.

\section{THEOREM: 3.6}

Let $\mathrm{A} \in \mathrm{C}^{\mathrm{nx} \mathrm{n}}$ be con s-normal circulant matrix then

(i) $\bar{A}$ is con s-normal circulant matrix

(ii) $\mathrm{A}^{\mathrm{S}}$ is con $\mathrm{s}-$ normal circulant matrix

(iii) $\mathrm{A}^{\ominus}$ is con s-normal circulant matrix

(iv) $\lambda \mathrm{A}$ is con s-normal circulant matrix (Where $\lambda$ is a non zero real no)

Proof:

$=\mathrm{A}^{\mathrm{S}} \bar{A}$

Let $\mathrm{A}$ be a con s-normal circulant matrix then $\mathrm{AA}^{\Theta}$

Proof of $(i) \mathrm{AA}^{\Theta}=\mathrm{A}^{\mathrm{S}} \bar{A}$

$$
\begin{aligned}
& \overline{\mathrm{AA}^{\theta}}=\overline{\mathrm{A}^{\mathrm{s}} \overline{\mathrm{A}}} \\
& \overline{\mathrm{A}^{\theta}} \overline{\mathrm{A}}=\mathrm{AA}^{\theta} \\
& \mathrm{A}^{\mathrm{T}} \overline{\mathrm{A}}=\mathrm{AA}^{\theta} \\
& \mathrm{AA}^{\theta}=\mathrm{A}^{\mathrm{s}} \overline{\mathrm{A}}
\end{aligned}
$$

Therefore $\bar{A}$ is con s-normal circulant matrix.

Proof of (ii) $\mathrm{AA}^{\Theta}=\mathrm{A}^{\mathrm{S}} \bar{A}$

$$
\begin{aligned}
\left(\mathrm{AA}^{\Theta}\right)^{\mathrm{S}} & =\left(\mathrm{A}^{\mathrm{S}} \bar{A}\right)^{\mathrm{S}} \\
\left(\mathrm{A}^{\Theta}\right)^{\mathrm{S}} \mathrm{A}^{\mathrm{S}} & =(\bar{A})^{\mathrm{S}}(\mathrm{A}) \\
(\bar{A}) \mathrm{A}^{\mathrm{S}} & =\mathrm{A}^{\Theta} \mathrm{A} \\
\mathrm{A}^{\mathrm{S}} \bar{A} & =\mathrm{AA}^{\Theta} \\
\mathrm{AA}^{\Theta} & =\mathrm{A}^{\mathrm{S}} \bar{A}
\end{aligned}
$$

Therefore $\mathrm{A}^{\mathrm{S}}$ is con s-normal circulant matrix.

Proof of (iii) $\mathrm{AA}^{\Theta}=\mathrm{A}^{\mathrm{S}} \bar{A}$

$$
\begin{aligned}
& \left(\mathrm{AA}^{\Theta}\right)^{\Theta}=\left(\mathrm{A}^{\mathrm{S}} \bar{A}\right)^{\Theta} \\
& \left(\mathrm{A}^{\Theta}\right)^{\Theta} \mathrm{A}^{\Theta}=(\bar{A})^{\Theta}\left(\mathrm{A}^{\mathrm{S}}\right)^{\Theta} \\
& \mathrm{AA}^{\Theta}=\mathrm{A}^{\mathrm{S}} \bar{A} \mathrm{~S} \\
& \mathrm{AA}^{\Theta}=\mathrm{A}^{\mathrm{S}} \bar{A}
\end{aligned}
$$

Therefore $\mathrm{A}^{\Theta}$ is con s-normal circulant matrix

Proof of (iv) $\mathrm{AA}^{\Theta}=\mathrm{A}^{\mathrm{S}} \bar{A}$

$$
\lambda^{2} \mathrm{AA}^{\Theta}=\lambda^{2} \mathrm{~A}^{\mathrm{S}} \bar{A}
$$

$$
(\lambda \mathrm{A})(\lambda \mathrm{A})^{\Theta}=\left(\lambda \mathrm{A}^{\mathrm{S}}\right)(\lambda \bar{A}) \text { where } \lambda=\lambda^{\Theta}
$$

Therefore $\lambda \mathrm{A}$ is con s-normal circulant matrix

\section{THEOREM: 3.7}

Let $A \in C^{n x n}$ and $A^{-1}$ be an inverse of $A$ then $A$ is con $\mathrm{s}-$ normal circulant matrix iff $\mathrm{A}^{-1}$ is con s-normal circulant matrix.

\section{Proof:} $=\mathrm{A}^{\mathrm{S}} \bar{A}$

To prove $\mathrm{A}^{-1}$ is con s-normal circulant matrix. We will show that $\left(\mathrm{A}^{-1}\right)\left(\mathrm{A}^{-1}\right)^{\Theta}=\left(\mathrm{A}^{-1}\right)^{\mathrm{S}}\left(\bar{A}^{-1}\right)$

$$
\text { Now } \begin{aligned}
\mathrm{A} \mathrm{A}^{\Theta} & =\mathrm{A}^{\mathrm{S}} \bar{A} \\
\left(\mathrm{AA}^{\Theta}\right)^{-1} & =\left(\mathrm{A}^{\mathrm{S}} \bar{A}\right)^{-1} \\
\left(\mathrm{~A}^{\Theta}\right)^{-1} \mathrm{~A}^{-1} & =(\bar{A})^{-1}\left(\mathrm{~A}^{\mathrm{S}}\right)^{-1} \\
\left(\mathrm{~A}^{-1}\right)^{\Theta}\left(\mathrm{A}^{-1}\right) & =\overline{\left(A^{-1}\right)}\left(\mathrm{A}^{-1}\right)^{\mathrm{S}} \\
\mathrm{A}^{-1}\left(\mathrm{~A}^{-1}\right)^{\Theta} & =\left(\mathrm{A}^{-1}\right)^{\mathrm{S}} \sqrt{\left.A^{-1}\right)}
\end{aligned}
$$

Therefore $\mathrm{A}^{-1}$ is con s-normal circulant matrix.

Let as assume that, $\mathrm{A}^{-1}$ is con s-normal circulant matrix.

To prove $\mathrm{A}$ is con s-normal circulant matrix. We will show that $\mathrm{A}^{\Theta}=\mathrm{A}^{\mathrm{S}} \bar{A}$

$$
\text { Now } \begin{aligned}
\mathrm{A}^{-1}\left(\mathrm{~A}^{-1}\right)^{\Theta} & =\left(\mathrm{A}^{-1}\right)^{\mathrm{S}} \sqrt{\left.A^{-1}\right)} \\
{\left[\mathrm{A}^{-1}\left(\mathrm{~A}^{-1}\right)^{\Theta}\right]^{-1} } & =\left[\left(\mathrm{A}^{-1}\right)^{\mathrm{S}}\left(\overline{A^{-1}}\right)\right]
\end{aligned}
$$




$$
\begin{aligned}
\mathrm{A}^{\Theta}\left(\mathrm{A}^{-1}\right)^{-1} & =\bar{A} \mathrm{~A}^{\mathrm{S}} \\
\mathrm{A}^{\Theta} \mathrm{A} & =\bar{A} \mathrm{~A}^{\mathrm{S}} \\
\mathrm{A} \mathrm{A}^{\Theta} & =\mathrm{A}^{\mathrm{S}} \bar{A}
\end{aligned}
$$

Therefore A is con s-normal circulant matrix.

\section{THEOREM: 3.8}

Let $\mathrm{A} \in \mathrm{C}^{\mathrm{nx} \mathrm{n}}$ and $\mathrm{A}^{\dagger}$ be the Moore Penrose inverse of $\mathrm{A}$ then $\mathrm{A}$ is con s-normal circulant matrix iff $\mathrm{A}^{\dagger}$ is con snormal circulant matrix

\section{Proof:}

Let $\mathrm{A}$ be a con s-normal circulant matrix then $\mathrm{AA}^{\Theta}=\mathrm{A}^{\mathrm{S}} \bar{A}$

To prove $\mathrm{A}^{\dagger}$ is con s-normal circulant matrix. We will show that $\left(\mathrm{A}^{\dagger}\right)\left(\mathrm{A}^{\dagger}\right)^{\Theta}=\left(\mathrm{A}^{\dagger}\right)^{\mathrm{S}}\left(\bar{A}^{\dagger}\right)$

Now

$$
\begin{aligned}
\mathrm{A} \mathrm{A}^{\Theta} & =\mathrm{A}^{\mathrm{S}} \bar{A} \\
\left(\mathrm{AA}^{\Theta}\right)^{\dagger} & =\left(\mathrm{A}^{\mathrm{S}} \bar{A}\right)^{\dagger} \\
\left(\mathrm{A}^{\Theta}\right)^{\dagger} \mathrm{A}^{\dagger} & =(\bar{A})^{\dagger}\left(\mathrm{A}^{\mathrm{S}}\right)^{\dagger} \\
\left(\mathrm{A}^{\dagger}\right)^{\Theta} \mathrm{A}^{\dagger} & =\left(\bar{A}^{\dagger}\right)\left(\mathrm{A}^{\dagger}\right)^{\mathrm{S}} \\
\mathrm{A}^{\dagger}\left(\mathrm{A}^{\dagger}\right)^{\Theta} & =\left(\mathrm{A}^{\dagger}\right)^{\mathrm{S}}\left(\bar{A}^{\dagger}\right)
\end{aligned}
$$

herefore $\mathrm{A}^{\dagger}$ is con s-normal circulant matrix.

Let as assume that, $\mathrm{A}^{\dagger}$ is con s-normal circulant matrix.

To prove $\mathrm{A}$ is con s-normal circulant matrix. We will show that $\mathrm{AA}^{\Theta}=\mathrm{A}^{\mathrm{S}} \bar{A}$

Now

$$
\begin{gathered}
\mathrm{A}^{\dagger}\left(\mathrm{A}^{\dagger}\right)^{\Theta}=\left(\mathrm{A}^{\dagger}\right)^{\mathrm{S}}\left(\bar{A}^{\dagger}\right) \\
{\left[\mathrm{A}^{\dagger}\left(\mathrm{A}^{\dagger}\right)^{\Theta}\right]^{\dagger}=\left[\left(\mathrm{A}^{\dagger}\right)^{\mathrm{S}}\left(\bar{A}^{\dagger}\right)\right]^{\dagger}} \\
\mathrm{A}^{\Theta}\left(\mathrm{A}^{\dagger}\right)^{\dagger}=\left(\bar{A}^{\dagger}\right) \mathrm{A}^{\mathrm{S}} \\
\mathrm{A}^{\ominus} \mathrm{A}=\bar{A} \mathrm{~A}^{\mathrm{S}} \\
\mathrm{A} \mathrm{A}^{\Theta}=\mathrm{A}^{\mathrm{S}} \bar{A}
\end{gathered}
$$

Therefore A is con s-normal circulant matrix.

\section{IV.CONCLUSION}

The s-normal circulant and con-s-normal circulant matrices defined and proof of structure theorem is shown by example.
We can find the determinant, inverse and some properties of the s-normal circulant matrices through the result.In future the various circulant matrices similar to unitary circulant, polynomial circulant matrices may be consider this study

\section{REFERENCES}

[1]. Elumalai.N, Rajesh kannan. K," k-symmetric Circulant, ssymmetric Circulant and s- k symmetric Circulant Matrices", Journal of Ultra Scientist of Physical Sciences, Vol.28(6),pp.322327 (2016).

[2]. Elumalai.N, Rajesh kannan. K," k-hermitian Circulant, s-hermitian Circulant and s- k hermitian Circulant Matrices",Int. Journal of Mathematics \& Statistics Invention, Vol.4(10), pp.22-28(2016)

3]. Elumalai.N, Rajesh kannan. K," Some Properties of k-normal Circulant and con-k normal Circulant Matrices" Int. Journal of Pure and Applied Mathematics, Vol 113 (10), 2017, 308 316(2017)

[4] Febbender.H., Ikramov.H,"Conjucate Normal Matrices: A survey," Linear Algebra and Appl.Vol.429 (2008).

[5].Hong-ping,M.A., Zheng-ke, MIAO, and Jiongsheng,L.I.,2008,"Generalized normal Matrix",Appl.Math.J.Chinese univ.,23(2),pp.240-244.

[6]. Horn, R.A., and Johnson,C.R., ,Matrix Analysis,Cambridge:Cambridge University press, Pp 79482(1985)

[7]. Krishnamoorthy. S, Vijayakumar. R, On s-normal matrices, J.of Analysis and Computation, Vol 15, No 2, 2009

[8].Krishnamoorthy.S.and Subash.R."On k-normal matrices "Int.J.of Math.Sci.\&Engg.Appls Vol.5(2009).

[9]. Krishnamoorthy. S, Gunasekaran.K, Arumugam.K," On con-k normal matrices" Int.J.of Current Research.Vol.4 (1).167- 169

[10].Penrose ,"A Generalized inverses for matrices"Proc.Cambridge Philos.soc.,Vol 51,406-413(1955).

\section{AUTHOR PROFILE:}

Elumalai. $\boldsymbol{N}$ serves as a Professor in Mathematics in A.V.C College, affiliated to Bharathidasan University. He has 29 years of experience in teaching and research in the field of Mathematics. He secured his Master and Master of Philosophy degrees from Madras University and Ph.D., from Government Arts College, Kumbakonam. He has published research papers in reputed national and international journals. He is presently guiding M.Phil., and Ph.D., scholars and produced M.Phil scholars

Rajesh kannan .K is presently working as Bachelor of teacher in Sri K.G.S Hr.Sec.School, Aduthurai, kumbakonam.He has 7 years of teaching experience in Engineering college. He earned Bachelor, Master and Master of philosophy degrees in the field of mathematics from bharathidasan university.He has qualified SET-UGC in Tamiladu.He has published three research papers and at present he has pursuing his research programme leading to Ph.D., degree in the field of circulant matrices. 